\author{
Mirko Đelošević \\ Ljiljana Vukajlov \\ Goran Tepić \\ Aleksandra Milinković \\ Dijana Brkljač
}

https://doi.org/10.21278/TOF.42204

ISSN 1333-1124

eISSN 1849-1391

\title{
DESIGN OF COLUMNS IN TERMS OF STABILITY
}

\begin{abstract}
Summary
In this paper, we investigate global and local stability of columns with an open and a closed thin-walled cross section. The proposed model of global stability is made of two deformable elements connected with an elastic joint. Stiffness of the elastic joint represents local discontinuity of the cross section of the column caused by the loss of stability of individual plates. The model of local stability of the columns is conceptualized on the principle of continuity of individual plates of the cross section. The coefficients of local buckling are defined as geometric parameters of the column with a rectangular hollow section (RHS) and a U-shaped cross section. The dominant parameters that influence the interactive behaviour of local and global buckling are the slenderness of plates and the column as a whole. The basic function of the developed models is to identify the stability mechanism in terms of better estimation of the critical force and higher load capacity.
\end{abstract}

Key words: $\quad$ buckling; plate; cross section; stability; column

\section{Introduction}

Design of support structures requires an analysis of stability of all elements that are subjected to load. The phenomenon of stability of the columns under load encompasses a complex mechanism of the interactive behaviour of local and global buckling [1]. The largest number of the here presented papers analyse the behaviour of a structure in terms of global stability. Buckling modes of columns under a load depend on the boundary conditions at the ends and on geometric imperfections. Models of buckling of a column with elastic supports according to dimensionless parameters, which represent rotational and translational constraints, as well as the factors of effective lengths are considered by Adman and Saidani [2]. An analysis of the global stability of columns with a rectangular cross section, eccentric supported by another element is carried out by Zang and Tong on a example of light supporting structures [3]. The proposed analytical model of this investigation is applicable to the analysis of geometrical imperfections and shows that the size of eccentricity and stiffness of the supported element are key parameters in defining the critical buckling force. Experimental and numerical research into flexural and lateral-torsional mode of buckling of an open thin-walled column with fixed supported ends and a $\mathrm{C}$-shaped cross section is 
presented by Gunalan and Mahendran [4]. It is shown that the currently applicable standards for the assessment of the resistance of elements to buckling, AS/NZS 4600 [5] and AISI-NAS [6], are substantially underestimated when the ends of the column are fixedly supported. Improvement of existing standards is carried out by using sophisticated numerical methods, such as CUFSM [7] and experimental testing. Experimental results for columns with a rectangular cross section show that the interaction between local and global buckling has a significant adverse effect on their resistance [8-9]. These papers have established a good correlation of the conducted tests for simultaneous occurrence of local and global buckling with the direct strength method (DSM), which is integrated into the standard procedures AISINAS and EC 3 [10].The buckling curve that defines the resistance of columns according to Eurocode 3 Part 1.4 (EN 3) provides a fairly conservative approach in the zone of interactive behaviour of local and global buckling which is not the case with the application of the DSM procedure [11]. In recent years, a large number of studies on stability of columns indicate the importance of local buckling analysis. The reason for this is the fact that in the thin-walled cross section the occurrence of local buckling initiates a global stability loss. In this respect, research on the stability of columns with a thin-walled closed cross section obtained by modifying the basic square shape was carried out in [12]. It was found that $\mathrm{L}, \mathrm{T}$ and + forms provide increased resistance to buckling of the columns by $10 \%, 50 \%$ and $90 \%$, respectively, in relation to the basic form, the RHS, as a result of a higher effective width $b_{\text {eff }}$ i.e. the effective cross-sectional area $A_{\text {eff }}$ [13]. The determination of the effective width and its impact on the stability of the steel box-shaped column is presented by Shanmugam et al. [14]. Higher values of these parameters provide a greater reserve of carrying capacity of columns after the occurrence of elastic local buckling or in the process of post-buckling of the plates [15]. In addition to the cross-sectional shape, the increase in the effective width of the plate of which the column is made can be achieved by installing longitudinal stiffeners [16]. Longitudinal stiffeners significantly affect local buckling so that it is reduced, but on the other hand can cause distortion of the cross section or distortional buckling [17]. The cross sections with stiffeners along the free edge of the plate elements (e.g. C, Z, $\Omega$ and other profiles) are particularly sensitive to distortion, which affects the interaction of local, distortional and global buckling [18]. The theoretical formulation of distortional buckling is presented by Kesti and Davies [19] on an example of C profiles, which is an integral part of the standards AS/NZS 4600. The assessment of resistance of open thin-walled columns in the distortional buckling mode using current applicable standards is discussed by $\mathrm{He}$ and Zhou [20]. According to the literature the research on local buckling columns is exclusively related to partial analysis of the plate with boundary conditions which approximately represent interactive influence of other elements of the cross section. The critical elastic buckling force of the combined loaded rectangular plate was defined by Shahabian and Roberts [21]. The behaviour of a plate with two fixed and two elastically supported edges was analysed in a recent study, published by Cai and Long [22]. Analytical solutions in the analysis of local buckling are particularly important because they provide more complete identification of the mechanism of stability of elements of the cross section [23-24].

\section{Models of global and local buckling of columns}

Global buckling manifests itself in the loss of stability of the column due to insufficient resistance of the cross section to the load transfer. This mode of buckling has long been known and represents an inevitable part of the analysis of columns. On the other hand, thinwalled cross sections which are sensitive to the occurrence of local buckling are used for a more rational design of columns. Open cross sections (e.g. U, C, Z, etc.) are characterized by low torsional stiffness with pronounced effects of distortional buckling (distortion of the cross section). It should be noted that distortion may occur in the closed cross section but the effect 
is less pronounced compared to open cross sections. Models of the stability of columns could be classified into two groups: a) models of global stability (based on the resistance of the entire cross-section) and b) models of local distortional stability (resistance of plates of the cross section). These models will be presented in the following sections of the paper on an example of an open and a closed cross section with special reference to the definition of the buckling coefficient and the critical buckling stress.

\subsection{Global buckling of the column}

The key parameters which have a direct impact on the occurrence of global buckling are related to the length of the column, the load and the shape of the cross section. Length and load are characteristics of the bearing structure which can not be significantly affected. The greatest opportunities for structural improvements regarding the reduction of sensitivity to the occurrence of buckling comes from a favourable choice of the cross-sectional shape [12]. This fact is especially significant when one takes the interactive relationship between the local and the global buckling into account. Optimally designed structures have a task to carry heavier load without significantly increasing their weight. Thus, columns have to be designed so that the cross section has approximately the same resistance to the loss of stability over the whole of its length $L$. This requires the use of variable cross section of the column, in some cases the rationalization of mass is achieved by creating perforated holes, although the existence of holes in the structure is often a construction requirement.

On the other hand, these structural requirements and the occurrence of local buckling on the elements of the cross section lead to a reduction in the effective cross-sectional area $A_{\text {eff, }}$, thereby a reduction in the stability of the column. Therefore, the need to develop models of global stability with $i$-th number of discrete elements mutually connected with $(i+1)$ springs of the bending stiffness $c_{i+1}$ and $(i+1)$ springs of the torsion stiffness $C_{i+1}$ arises. Zones of discontinuity of the cross section of the column correspond with the increase or the decrease in their bending stiffness compared to the rest of the column. The model of global stability with two discrete elastic elements is presented in Fig. 1b. The total potential energy of $T_{i}$ for the $i$-th deformable element with elastic connections is the sum of the strain energy $U_{i}$ and the external work $V_{i}$, which is a consequence of the action of the external force $F$.

$$
\begin{aligned}
& \delta T=U_{i}+V_{i}=0 \Rightarrow \\
& \frac{1}{2} C_{j}\left(y_{j}^{\prime}\right)^{2}+\frac{1}{2} c_{j}\left(y_{j}\right)^{2}+\frac{1}{2} C_{j+1}\left(y_{j+1}^{\prime}\right)^{2}+\frac{1}{2} c_{j+1}\left(y_{j+1}\right)^{2}+\frac{1}{2} E I_{i} \int_{0}^{L_{i}}\left(y^{\prime \prime}\right)^{2} d x-\frac{1}{2} F \int_{0}^{L_{i}}\left(y^{\prime}\right)^{2} d x=0
\end{aligned}
$$

The necessary and sufficient conditions which provide that $\delta T=0$ have the following form [29]:

$$
E I_{y}^{(I V)}+F y^{\prime \prime}=0
$$

where (the parameter $y_{j}$ represents the lateral movement of nodes, $j=1,2,3$ ):

$$
\begin{aligned}
& \left(C_{j+1} y_{j+1}^{\prime}+E I_{i} y_{j+1}^{\prime \prime}\right) \delta y_{j+1}^{\prime}=0 \\
& \left(C_{j} y_{j}^{\prime}-E I_{i} y_{j}^{\prime \prime}\right) \delta y_{j}^{\prime}=0 \\
& \left(c_{j+1} y_{j+1}-E I_{i} y_{j+1}^{(I I I)}-F y_{j+1}^{\prime}\right) \delta y_{j+1}^{\prime}=0 \\
& \left(c_{j} y_{j}+E I_{i} y_{j}^{(I I I)}+F y_{j}^{\prime}\right) \delta y_{j}^{\prime}=0
\end{aligned}
$$

The general solution to equation (9) has the following form:

$$
y=A \sin \phi_{i} x+B \cos \phi_{i} x+C x+D, \phi_{i}^{2}=\frac{F}{E I_{i}}
$$


The integration constants $A, B, C$ and $D$ are determined from the system which defines the boundary conditions on the ends of the column. This system is written in a compact form as follows:

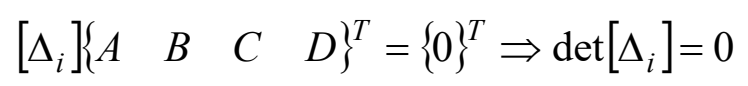

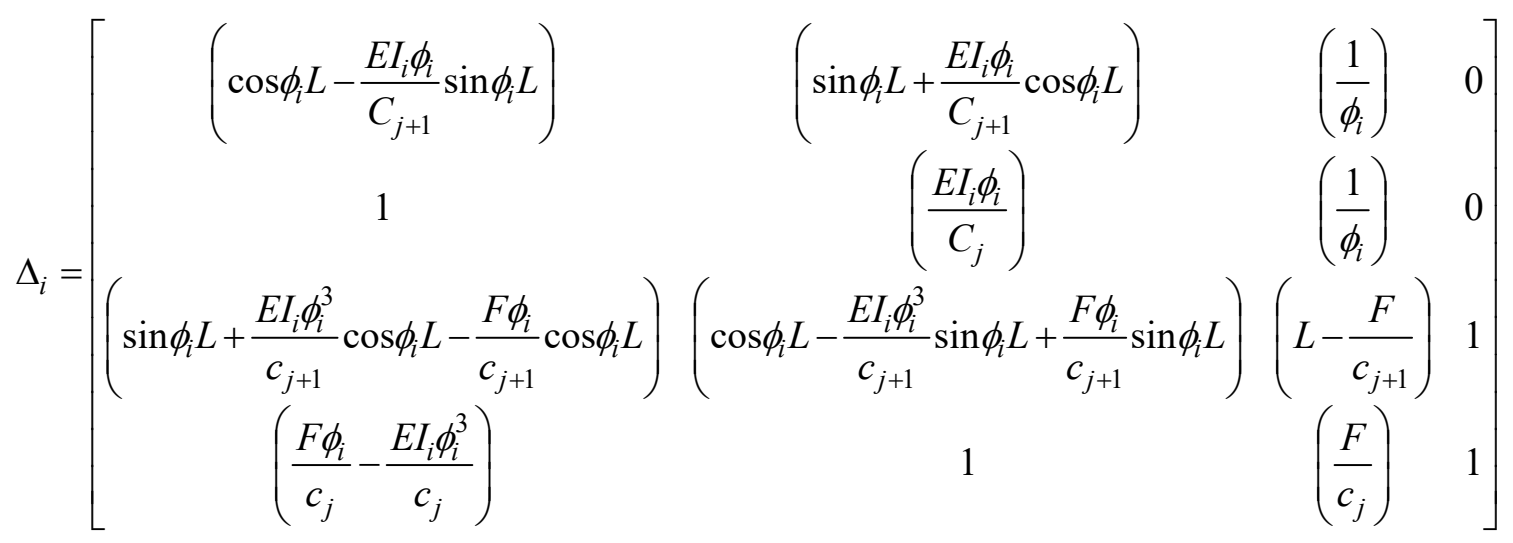

\subsection{Local buckling of the column}

The zones of local buckling of the cross section correspond with the reduction of the effective cross-sectional area regardless of the fact that the material in these zones physically exists (this refers to ineffective zones). The stability of the columns formed of plates (e.g. by cold forming or welding) requires an interactive analysis of individual elements, i.e. the plates. In this regard, it is necessary to perform a structural decomposition of the column on individual elements (plates) of the same length, whereas in a general case elements are of different widths and thicknesses. The theoretical basis for identification of local stability of columns includes a partial analysis of individual elements of which a cross section is formed. Depending on the type of cross section of the column, various contour conditions are imposed that significantly influence the buckling of certain plates and have an indirect impact on the stability of the overall cross section. The end edges of the plates from which the column is formed are most often connected to the base plates, which correspond to a completely fixed support. However, the analysis of the local stress of box girders presented in study [26] shows that the conditions of a fixed supported girder have influence on the behaviour of long plates only in their immediate vicinity. The differential equation of the elastic behaviour of the plate according to [27-28] reads as:

$$
\frac{\partial^{4} w}{\partial x^{4}}+2 \frac{\partial^{4} w}{\partial x^{2} y^{2}}+\frac{\partial^{4} w}{\partial y^{4}}+\frac{\sigma_{x} t}{D} \frac{\partial^{2} w}{\partial x^{2}}=0, \quad D=\frac{E t^{3}}{12\left(1-v^{2}\right)}
$$

The general solution that corresponds to differential equation (1) has the following form [28]:

$w=\sum_{m=1}^{\infty}\left\{A_{1} \cosh \xi y+A_{2} \sinh \xi y+A_{3} \cos \eta y+A_{4} \sin \eta y\right\} \sin \frac{m \pi x}{a}$

where: $\xi=\sqrt{\left(\frac{m^{2} \pi^{2}}{a^{2}}\right)+\left(\frac{\sigma_{x} t}{D} \frac{m^{2} \pi^{2}}{a^{2}}\right)^{\frac{1}{2}}} ; \eta=\sqrt{-\left(\frac{m^{2} \pi^{2}}{a^{2}}\right)+\left(\frac{\sigma_{x} t}{D} \frac{m^{2} \pi^{2}}{a^{2}}\right)^{\frac{1}{2}}}$

The coefficients $A_{1}, A_{2}, A_{3}$ and $A_{4}$ are determined from the boundary conditions that define the state of the plate along the longitudinal edges.

$D$ is the flexural rigidity of the plate and $\sigma_{x}$ is the normal stress in the plane of the plate. 


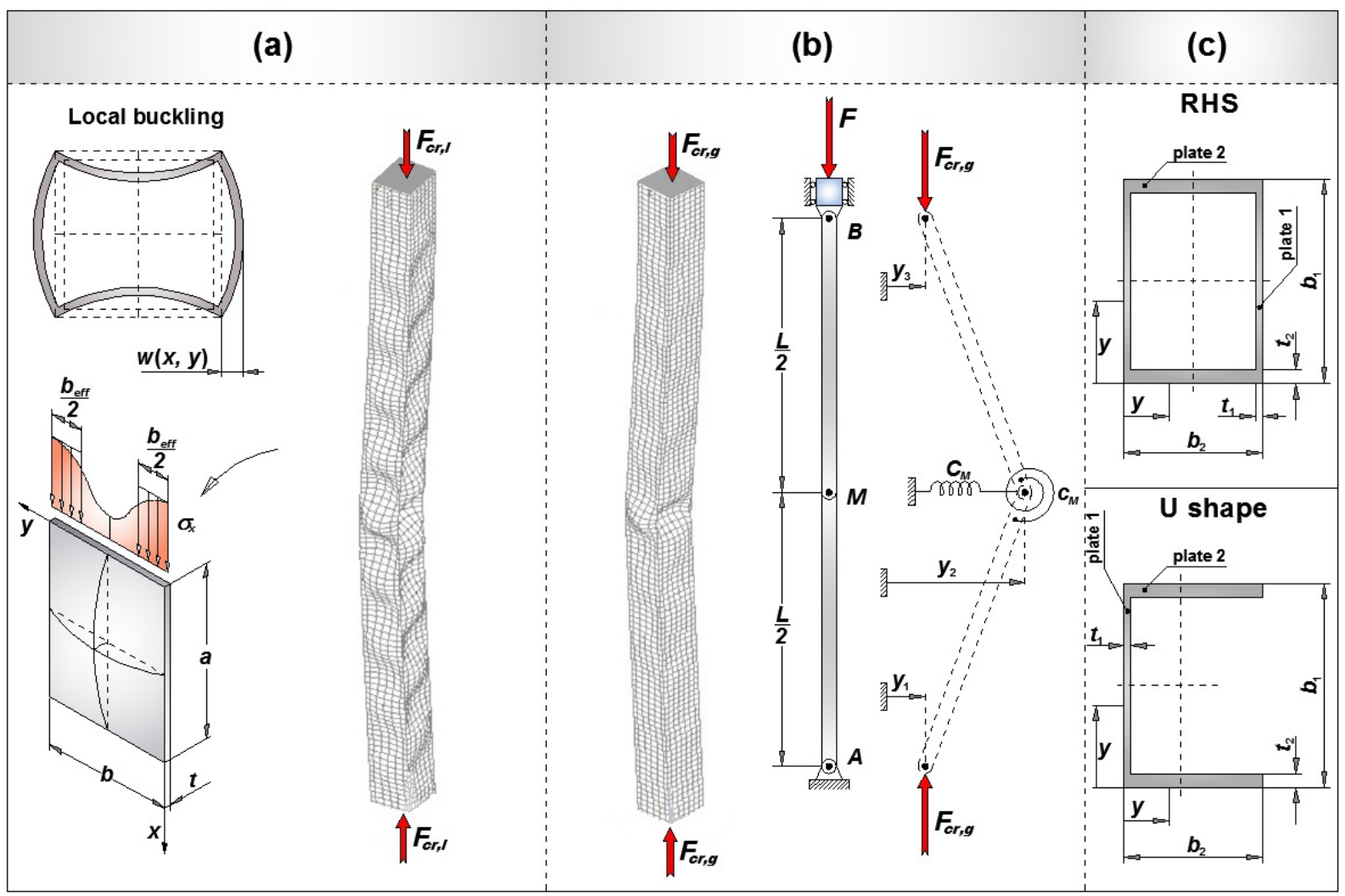

Fig. 1 Models of local buckling (a), global buckling (b) and considered cross-sections (c)

The critical force of the local buckling $F_{c r, l}$ has a value which is different from the critical force of the global buckling $F_{c r, g}$, regardless of the fact that the column in both cases has the same length and cross section. Global buckling occurs after the loss of stability in the most exposed section of the column (the middle section of a - simply supported column), while local buckling is characterized by the appearance of waves along the whole length of the column. Types of boundary conditions and characteristic equations, depending on the type of cross section of the column and the conditions of the supported individual plates of cross section, are presented in Tables 1 and 2. Closed cross sections (e.g., rectangular cross sections) are characterized by interactions with neighbouring plates through their two lateral edges. Due to the symmetry of cross section, boundary conditions need to be defined along two longitudinal lines of the plates (Table 1). The first line refers to the centre line of the plate, where changes in deflection and moment have extreme values. The second line corresponds to the line of the joint of two adjacent plates along which transverse displacement is prevented and continuity of the cross section is ensured [26]. The boundary conditions in the longitudinal direction (along the $x$-axis) correspond with the free supported plates, because slender plates are characterized by a large number of half waves which changes sign. The inflection point of the half wave is equivalent to a simply supported edge of the plate because deflections and bending moments along this line are characterized by the change of sign and have zero values (Fig. 1a). Open cross sections (e.g. U profiles) are characterized by the plates with free ends (flange profiles) which generate appropriate boundary conditions. These conditions are related to the formulation of transverse forces and bending moment along the free edge of the plate which must have a value of zero (Table 2). Boundary conditions for the rib profile are identical with formulations that are valid for a rectangular cross section. The condition of the stability of columns of different types of cross sections is defined by the characteristic equations $\mathrm{S}$ and $\mathrm{B}$, given in Tables 1 and 2. 
Table 1 Boundary conditions and equations for RHS Table 2 Boundary conditions and equations for U profile

\begin{tabular}{|c|c|c|c|}
\hline $\begin{array}{l}\text { Edge } y=0 \\
\text { simply supported } \\
\text { Edge } y=b / 2 \\
\text { in plane of symmetry }\end{array}$ & $\begin{array}{l}\text { Edge } y=0 \\
\text { built-in } \\
\text { Edge } y=b / 2 \\
\text { in plane of symmetry }\end{array}$ & $\begin{array}{l}\text { Edge } y=0 \\
\text { simply supported } \\
\text { Edge } y=b \\
\text { free }\end{array}$ & $\begin{array}{l}\text { Edge } y=0 \text { built-in } \\
\text { Edge } y=b \text { free }\end{array}$ \\
\hline$(w)_{y=0}=0$ & $(w)_{y=0}=0$ & $(w)_{y=0}=0$ & $(w)_{y=0}=0$ \\
\hline$\left(\frac{\partial^{2} w}{\partial y^{2}}+v \frac{\partial^{2} w}{\partial x^{2}}\right)_{y=0}=0$ & $\left(\frac{\partial w}{\partial y}\right)_{y=0}=0$ & $\left(\frac{\partial^{2} w}{\partial y^{2}}+v \frac{\partial^{2} w}{\partial x^{2}}\right)_{y=0}=0$ & $\left(\frac{\partial w}{\partial y}\right)_{y=0}=0$ \\
\hline$\left(\frac{\partial w}{\partial y}\right)_{y=\frac{b}{2}}=0$ & $\left(\frac{\partial w}{\partial y}\right)_{y=\frac{b}{2}}=0$ & $\left(\frac{\partial^{3} w}{\partial y^{3}}+v \frac{\partial 3 w}{\partial x^{2} \partial y}\right)_{y=b}=0$ & $\left(\frac{\partial^{3} w}{\partial y^{3}}+v \frac{\partial^{3} w}{\partial x^{2} \partial y}\right)_{y=b}=0$ \\
\hline$\left(\frac{\partial^{3} w}{\partial y^{3}}+\frac{\partial^{3} w}{\partial x^{2} \partial y}\right)_{y=\frac{b}{2}}=0$ & $\left(\frac{\partial^{3} w}{\partial y^{3}}+\frac{\partial^{3} w}{\partial x^{2} \partial y}\right)_{y=\frac{b}{2}}=0$ & $\left(\frac{\partial^{3} w}{\partial y^{3}}+(2-v) \frac{\partial^{3} w}{\partial x^{2} \partial y}\right)_{y=b}=0$ & $\left(\frac{\partial^{3} w}{\partial y^{3}}+(2-v) \frac{\partial^{3} w}{\partial x^{2} \partial y}\right)_{y=b}=0$ \\
\hline $\begin{array}{l}S=\alpha \beta\left(\gamma^{2}+\delta^{2}\right) \\
\cosh \frac{\alpha}{2} \cos \frac{\beta}{2}\end{array}$ & $B=\alpha \beta\left(\begin{array}{l}\alpha \sinh \frac{\alpha}{2} \cos \frac{\beta}{2}+ \\
\beta \cosh \frac{\alpha}{2} \sin \frac{\beta}{2}\end{array}\right)$ & $\begin{array}{l}S=-\left(\gamma^{2}+\delta^{2}\right) . \\
\cdot\left(\begin{array}{l}\beta \gamma^{4} \sinh \alpha \cos \beta- \\
-\alpha \delta^{4} \cosh \alpha \sin \beta\end{array}\right)\end{array}$ & $\begin{array}{l}B=2 \alpha \gamma \gamma^{2} \delta^{2}+ \\
+\alpha \beta\left(\gamma^{4}+\delta^{4}\right) \cosh \alpha \cos \beta+ \\
+\left(\beta^{2} \gamma^{4}-\alpha^{2} \delta^{4}\right) \sinh \alpha \sin \beta\end{array}$ \\
\hline
\end{tabular}

The parameters $\alpha, \beta, \gamma$ and $\delta$, represented in the characteristic equations, are given by the following expressions (the number of wavelength plates defines $m=1,2,3, \ldots$ ):

$$
\alpha=\sqrt{\left(\frac{m \pi^{2}}{\varepsilon}\right) \cdot\left(\sqrt{k}+\frac{m}{\varepsilon}\right)} ; \beta=\sqrt{\left(\frac{m \pi^{2}}{\varepsilon}\right) \cdot\left(\sqrt{k}-\frac{m}{\varepsilon}\right)} ; \gamma=\alpha^{2}-v\left(\frac{m^{2} \pi^{2}}{\varepsilon^{2}}\right) ; \delta=\beta^{2}-v\left(\frac{m^{2} \pi^{2}}{\varepsilon^{2}}\right)
$$

where $\varepsilon$ is the parameter of wavelength of local buckling of the rectangular plate which is defined as the relation of dimensions of the rectangular plates $(\varepsilon=a / b)$, according to Fig. 1a, while $k$ represents the coefficient of local buckling and $v$ is Poisson's ratio $(v=0.3)$.

Local buckling is related only to the loss of stability of individual elements of the cross section, without displacement of their built-in or free edges and distortion of the cross section (rotation of flanges in an open cross section). The rectangular cross section is composed of four plates which are connected to each other along the longitudinal edges in an inseparable unit. The interaction of the structural elements of the cross section is represented by boundary conditions along the joint lines which relate to the limitation of the lateral displacements as well as the condition of continuity as follows:

$$
\left(w_{p}\right)_{y=b_{p}}=0 ;\left(\frac{\partial w_{p+1}}{\partial y}\right)_{y=0}=\left(\frac{\partial w_{p}}{\partial y}\right)_{y=b_{p}} ;\left(w_{p+1}\right)_{y=b_{p+1}}=0 ;\left(\frac{\partial w_{p+2}}{\partial y}\right)_{y=0}=\left(\frac{\partial w_{p+1}}{\partial y}\right)_{y=b_{p+1}}
$$

where $k$ represents the number of plates from which the rectangular cross section is formed $(p=1,2,3,4)$. When the appropriate function of deflection $w_{p}(x, y)$ is applied to the boundary conditions of the system of plates (15), which are defined by (11), we obtain a system of four equations which are equivalent to the determinant of the system of order $4 \times 4$, which is necessary to define the stable equilibrium of the structural elements of the cross section. The determinant of the system mentioned above can be written in a compact form as follows [30]:

$$
\sum_{n=1}^{p} \frac{1}{\varphi_{n}^{3} \lambda_{n}}\left[\frac{S}{B}\right]=0
$$


where $\varphi_{n}\left(\varphi_{n}=t_{n} / t_{p}\right)$ and $\lambda_{n}\left(\varphi_{n}=b_{p} / b_{n}\right)$ are geometric parameters of the cross section; where $t$ is the thickness, $b$ is the width and $p$ is the number of characteristic plates. When applied to the two different plates connected along a common longitudinal edge, we obtain:

$$
\left[\frac{S}{B}\right]_{1}+\frac{1}{\varphi_{1}^{3} \lambda_{1}}\left[\frac{S}{B}\right]_{2}=0
$$

where $\varphi_{1}\left(\varphi_{1}=t_{1} / t_{2}\right)$ and $\lambda_{1}\left(\varphi_{n}=b_{2} / b_{1}\right)$ are characteristics of the RHS and the $\mathrm{U}-$ shaped cross section. After appropriate expressions for $S$ and $B$ from Table 1 have been introduced in (20) we obtain:

$$
\left[\frac{-\left(\gamma^{2}+\delta^{2}\right) \cosh \frac{\alpha}{2} \cos \frac{\beta}{2}}{\alpha \sinh \frac{\alpha}{2} \cos \frac{\beta}{2}+\beta \cosh \frac{\alpha}{2} \sin \frac{\beta}{2}}\right]_{1}+\frac{1}{\varphi_{1}^{3} \lambda_{1}}\left[\frac{-\left(\gamma^{2}+\delta^{2}\right) \cosh \frac{\alpha}{2} \cos \frac{\beta}{2}}{\alpha \sinh \frac{\alpha}{2} \cos \frac{\beta}{2}+\beta \cosh \frac{\alpha}{2} \sin \frac{\beta}{2}}\right]_{2}=0
$$

The condition (16) corresponds to the boundary condition of stability of the thin-walled rectangular cross section when local buckling occurs. The relevant length of the column to analyse local buckling is assessed in relation to the maximum cross-sectional dimension $(a \approx b)$. This length is exactly determined by the parameter $\varepsilon$ in the conditions of local stability (18). Boundary conditions of open cross sections are more complex because of the plates with free edges (flange of the U-shaped cross section) or elastically supported edges (flange of the C-shaped cross section). The boundary conditions (19a) and (19b) refer to the rib of the $U$ profile and they are equivalent to the previous case, while the formulations (19c) and (19d) are characterized by the boundary conditions of their flanges. Indices 1 and 2 refer to the plate cross section with different widths.

$$
\left(w_{j}\right)_{y=b_{j}}=0 ;\left(\frac{\partial w_{j+1}}{\partial y}\right)_{y=0}=\left(\frac{\partial w_{j}}{\partial y}\right)_{y=b_{j}} ;\left(\frac{\partial^{3} w_{j+1}}{\partial y^{3}}+(2-v) \frac{\partial^{3} w_{j+1}}{\partial x^{2} \partial y}\right)_{y=b_{j+1}}=0 ;\left(\frac{\partial^{2} w_{j+1}}{\partial y^{2}}+v \frac{\partial^{2} w_{j+1}}{\partial x^{2}}\right)_{y=b_{j+1}}=0
$$

Except by local buckling, the open cross section is characterized by distortion of the cross section (distortional buckling), especially of the profile with strengthened edges, such as the $\mathrm{C}$ profile. Distortional mode occurs in columns whose approximate length is in the range of $10 a<L_{d i s}<100 a$. Lower values $L_{d i s}$ affect the introduction of local distortion, whereas higher values of $L_{d i s}$ cause interaction between distortional buckling and flexural-torsional buckling. The length of the column at which purely distortional buckling occurs depends on the type of the load and the cross sectional shape. This length must be large enough to avoid local buckling and significantly smaller than the actual length of column $L$ in order to avoid interaction with global buckling. Analogously to the previous case, replacing the function of deflection (2) in the boundary conditions (19) leads to the equation of local (distortional) stability (20) of elements of the cross section which is related to the U profile (the same form holds for the $\mathrm{C}$ profile, but with different coefficients).

$$
\begin{aligned}
& {\left[\frac{-\left(\gamma^{2}+\delta^{2}\right) \cosh \frac{\alpha}{2} \cos \frac{\beta}{2}}{\alpha \sinh \frac{\alpha}{2} \cos \frac{\beta}{2}+\beta \cosh \frac{\alpha}{2} \sin \frac{\beta}{2}}\right]_{1}+} \\
& +\frac{1}{\varphi_{1}^{3} \lambda_{1}} \cdot\left[\frac{-\left(\gamma^{2}+\delta^{2}\right) \times\left(\beta \gamma^{4} \sinh \alpha \cos \beta-\alpha \delta^{2} \cosh \alpha \sin \beta\right)}{2 \alpha \beta \gamma^{2} \delta^{2}+\alpha \beta\left(\gamma^{4}+\delta^{4}\right) \cosh \alpha \cos \beta+\left(\beta^{2} \gamma^{4}-\alpha^{2} \delta^{4}\right) \sinh \alpha \sin \beta}\right]_{2}
\end{aligned}
$$




\section{Discussion and verification of results}

The condition of global stability (14) implemented in the simply supported column (for supports $\mathrm{A}$ and $\mathrm{B}$ there is: $C_{1}=C_{3} \rightarrow \infty$ and $c_{1}=c_{3}=0$ ) with two deformable segments, which are connected with an elastic connection in the point $\mathrm{M}\left(C_{2}=C_{M}\right.$ and $\left.c_{3}=c_{M}\right)$, allows identification of the critical mode of stability caused by the occurrence of elastic local buckling (Fig. 1b). Flexural stiffness of the spring $c_{M}$ which prevents relative rotation of the elements of the column around the point $\mathrm{M}$ is given by $c_{M}=\mu E I / L$. Extensional stiffness of the spring $C_{M}$ limits the translational movement of the point $\mathrm{M}$ in relation to the initial position of the column and it is defined as $C_{M}=\psi E I / L^{3}$. When the conditions of the supported column are implemented, which is formed of two segments of the same lengths $(L / 2)$ mutually elastically connected according to Fig. $2 b$, the determinant (14) is reduced to the equation of global stability (21).

$$
\cos \left(\pi \sqrt{p_{N}}\right) \cdot\left[1-\frac{\pi^{2}}{\psi} p_{N}\right]-\sin \left(\pi \sqrt{p_{N}}\right) \cdot\left[\frac{1}{\pi \sqrt{p_{N}}}+\frac{\pi}{\mu} \sqrt{p_{N}}\left(1-\frac{\pi^{2}}{\psi} p_{N}\right)\right]=0
$$

where $p_{N}\left(p_{N}=F / F_{0}\right)$ represents the normalized critical force of global buckling. Euler's (critical) buckling load for a simply supported column amounts to $F_{0}=\pi^{2} E I / L^{2}$. The column is in the position of stable equilibrium if it satisfies equation (21), while $p_{N, c r}$ is the value that corresponds to this stable state. The critical force of the global buckling $F_{c r}$ is determined by the normalized critical force $p_{N, c r}$ and the reference critical buckling load of the columns which correspond to the model of the simply supported column $F_{0}$. The $\mu$ and $\psi$ parameters are non-dimensionless coefficients that define the elasticity of the connection at point $\mathrm{M}$ of the column and their value depends on the type and the dimensions of the cross section, i.e. the effective width $b_{\text {eff }}$ [13]. The dependence of the normalized force $p_{N}$ according to parameters $\mu$ and $\psi$ is illustrated in Fig. 2. For the value $\mu=10$, the mathematical model (21) is equivalent to Euler's critical force. The lower value of the coefficient $\mu$ tends to be constant when $\psi$ grows. On the other hand, the increase in the parameter $\mu$ increases the resistance to the bending of the column at point $\mathrm{M}$, allowing for a higher critical force which degressively increases with an increase of parameter $\psi$. The critical buckling stress, caused by the loss of global stability, is defined as:

$$
\sigma_{c r, e}=\frac{F_{c r}}{A}=\frac{p_{N} \pi^{2} E}{\lambda^{2}}<\sigma_{p e r}
$$

where $\sigma_{p e r}$ is a permissible value of the stress. The critical force of the elastic buckling, which is defined according to the criteria of global stability, is insufficient for a complete evaluation of the stability of the column as a whole. Global stability depends solely on the slenderness of column $\lambda$ which is defined as the ratio buckling length $L_{b u c k}$ and the minimum radius of gyration $r_{\text {min }}$. Column length $L$ has a significant impact on its stability, but this parameter is usually a construction condition and the possibility of its variation is limited to a considerable extent. Young's modulus of elasticity $E$ can be influenced by the choice of different types of materials, which is also a limiting factor. Therefore, the dominant parameter that affects the global stability of the column refers to the axial moment of inertia $I$. Rationality of the supporting structures is achieved by applying a thin-walled cross-section, which is characterized by the minimum mass, i.e. the ratio $A / I$. This means that with a small surface of the cross section $A$ a high resistance of the cross section to bending can be achieved, defined by the parameter $I$. If we observe a rectangular and a square shape of the same cross-sectional area, we can conclude that according to (22), a square cross section has greater stability. The increase in the axial moment of inertia $I$ results from larger cross-sectional dimensions $b \times b$ and reduced plate thickness $t$. An unfavourable ratio $t / b$ can initiate local buckling and failure of the column as a whole. 


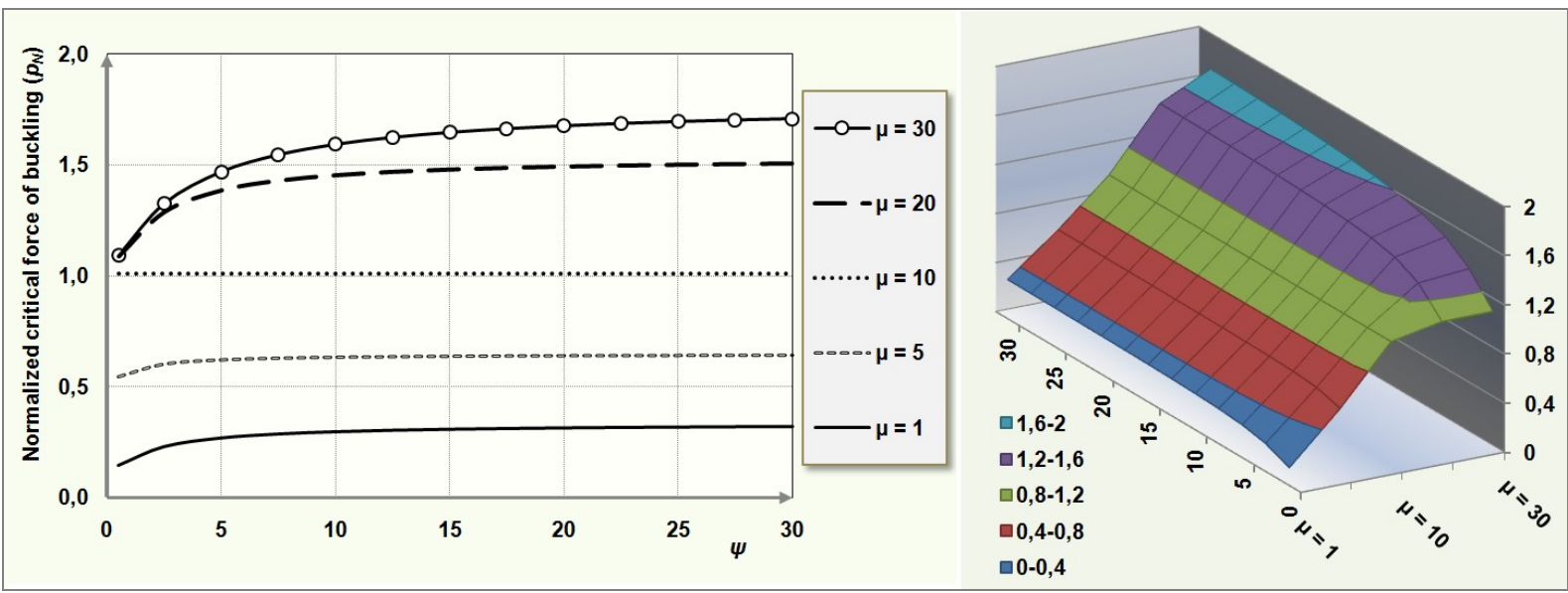

Fig. 2 Normalized critical buckling force as non-dimensionless parameters $p_{N}=f(\mu, \psi)$

The ratio of $t / b$ must be above the area of local buckling (Fig. 4, left). On the other hand, thin-walled cross sections result in the occurrence of a phenomenon of local buckling, which can induce instability regardless of the fact that the aspect of global buckling shows the state of stability of the column. Thus, the stability analysis of a column requires identification of interactive behaviour of local and global buckling. The mechanisms of local and global buckling are different one from another and the interactivity between these two phenomena is established through the cross section. Namely, the cross section provides the required stiffness of the column $E I$ in terms of bending resistance of the column. Certain flexural stiffness of the column is achieved by different cross sections, which is completely irrelevant from the aspect of the global stability model. However, the type and shape of the cross section have a great influence on the parameters of local buckling, as well as on the occurrence of the distortional mode of buckling. This assertion can be illustrated by an example of a closed and an open cross section (Fig. 3). The analysis of the local stability of the rectangular cross section shows that an increase in the width ratio $b_{2} / b_{1}$ causes a reduction in the coefficient of local buckling $k$. Definition of the local buckling coefficient $k$ is done with respect to the characteristic values $b_{2} / b_{1}=1(k=4)$. The cross sections most sensitive to local buckling are characterized by higher values of the parameter $t_{1} / t_{2}$ due to a decrease in the coefficient of the local buckling $k$ with the increase in $b_{2} / b_{1}$. In terms of local stability, the square shape is the optimal configuration of the column with a four-sided closed cross section. The wavelength of local buckling is defined by the parameter $\varepsilon$ and has a tendency of constant increase (by approximately linear dependence) as the parameter $b_{2} / b_{1}$ increases. The wavelength for the square cross section has a minimum value and amounts to $b\left(L_{b u c k}=b \rightarrow \varepsilon=1\right)$.
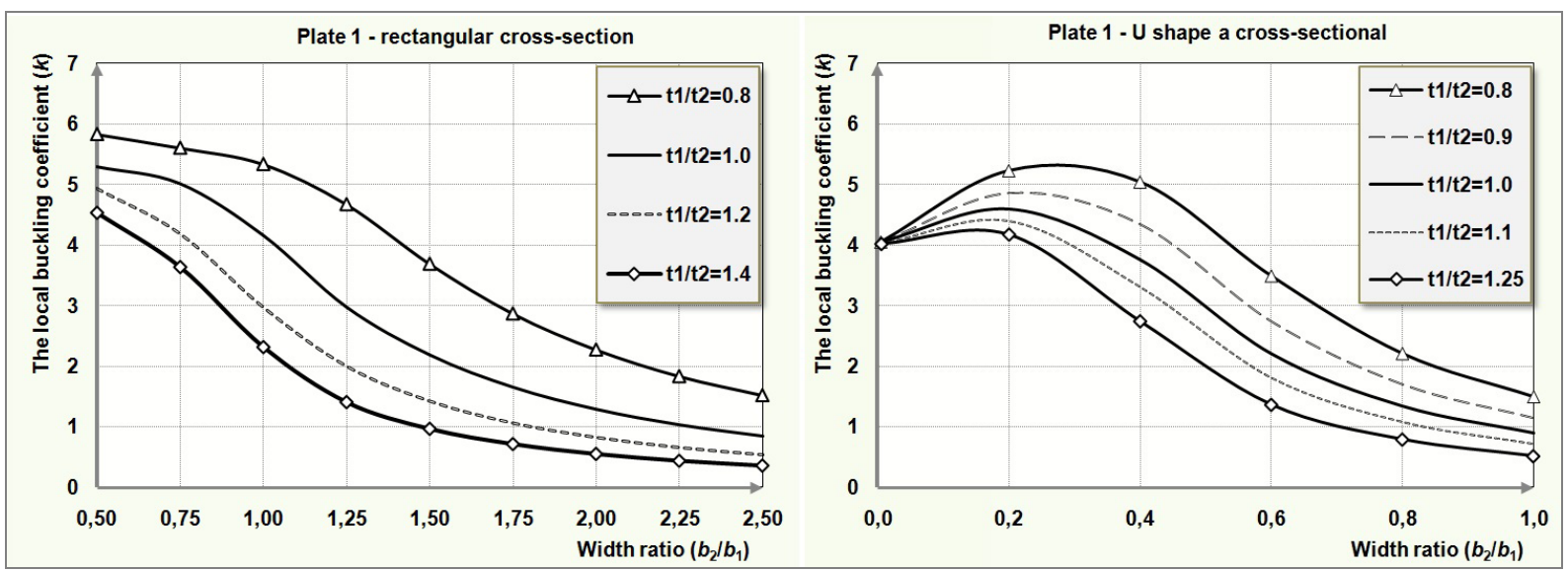

Fig. 3 Coefficient of local buckling $k=f\left(b_{2} / b_{1}\right)$ for rectangular cross-section (RHS) and U-shaped cross section 
The critical stress value caused by local buckling is defined as:

$$
\sigma_{c r, l}=\min \left\{\frac{k_{m} \pi^{2} E}{12\left(1-v^{2}\right)}\left(\frac{t_{m}}{b_{m}}\right)^{2}\right\}<\sigma_{p e r}
$$

where $m$ is the number of different plate elements of the cross section (e.g., for RHS and U profile, $m=2$ ). The critical force of local buckling corresponds to the minimum values of stress defined for each individual element of the cross section, according to the equation (23). Local stability of the U-shaped cross section is characterized by the curve of the local buckling coefficient $k=k\left(b_{2} / b_{1}\right)$, which is shown in Fig. 3 (right). If $t_{1} / t_{2}=1$, the maximum value of the local buckling coefficient is $k=4.593$ for the ratio of the width flange to the rib $b_{2} / b_{1}=0.2$. Accordingly, the optimum width of the flanges for the U-shaped profile in terms of local stability is $b_{2}=0.2 b_{1}$ ( $b_{1}$ is the width of the rib). The wavelength of buckling for Ushaped profiles is characterized by higher values of $b_{2} / b_{1}$ (relative to the RHS) as a consequence of the free edge of the flanges. Plate 1 of the U-shaped cross section refers to the rib profile (Fig. 1). The two main parameters of local stability include the buckling coefficient $k$ and the wavelength $\varepsilon$. Minimum values of the parameters $k$ and $\varepsilon$ that satisfy (18) for the RHS and (20) for the U-shaped profiles are authoritative for the analysis of local stability.
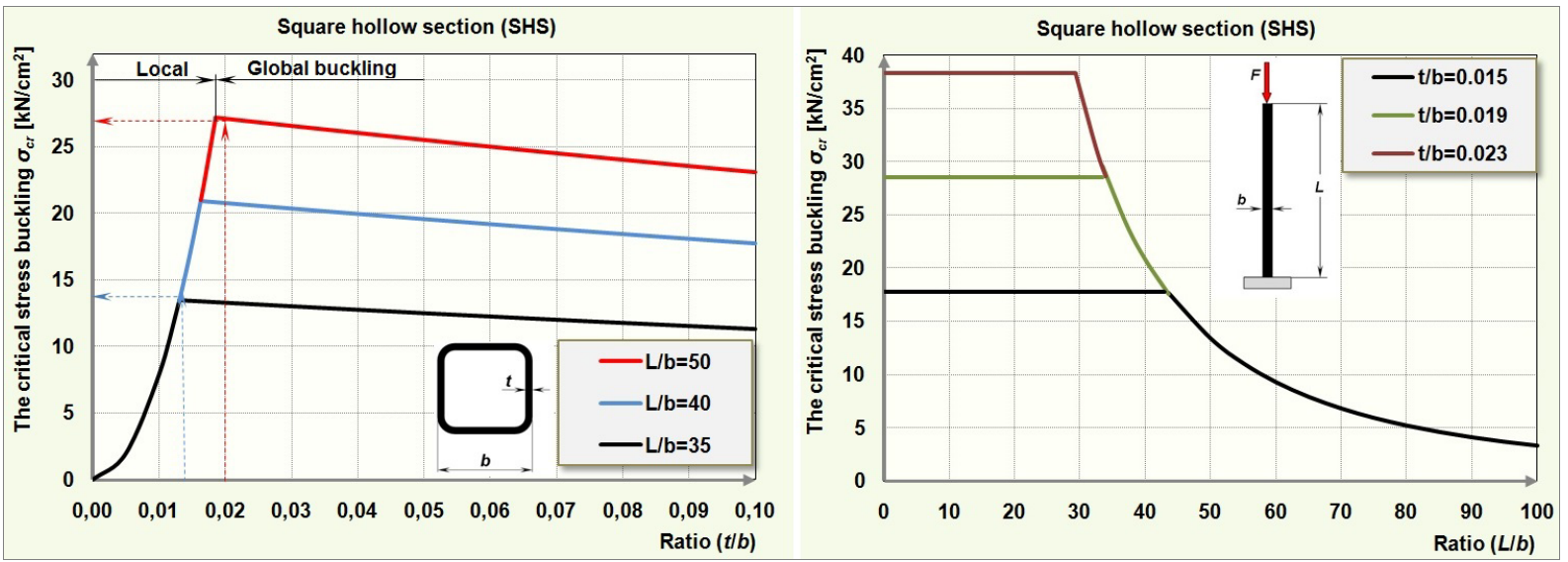

Fig. 4 Interaction between local and global buckling for square hollow section (SHS)

The interactive behaviour of local and global buckling of a thin-walled cross-section of a squared shape is shown in Fig. 4. The key geometrical sizes which lead to the interaction of local and global buckling refer to the parameters of $t / b$ and $L / b$. Plate thickness $t$ is characterized by local and global buckling, whereas the column length $L$ has only influence on global buckling. The dimension of the cross section $b$ is also a geometric parameter that has an influence on both models of buckling. By reducing the slenderness of the plate elements $t / b$ and increasing the slenderness of the column $L / b$, the critical stress of buckling $\sigma_{c r}$ moves from the area of local buckling towards the zone of global buckling (Fig. 4). The verification of the presented models was performed in accordance with EN 1993-1 [10] and experimental data [1]. A comparative analysis of the results for three columns of different lengths with square hollow sections (SHSs) is given in Table 3. The theoretical models show consistency with experimental data, while EN 1993-1 has a more conservative approach.

Table 3 Comparative analysis and verification of results

\begin{tabular}{cccccccccc}
\hline \multirow{2}{*}{ Case } & $\begin{array}{c}\text { Length } \\
{[\mathrm{mm}]}\end{array}$ & \multicolumn{2}{c}{ Slenderness } & \multicolumn{2}{c}{ Coefficients } & \multicolumn{2}{c}{ Maximum critical force $F_{c r}[\mathrm{kN}]$} & Deviation \\
\cline { 3 - 9 } & local & global & $t / b$ & $L / b$ & EN 1993-1 & Experimental & Theory & Exp/Th [\%] \\
\hline 1 & 2200 & 0.85 & 0.35 & 0.22 & 8.50 & 1891 & 2139 & 2204 & 2.95 \\
\hline 2 & 3700 & 0.85 & 0.55 & 0.22 & 14.25 & 1803 & 2065 & 2204 & 6.30 \\
\hline 3 & 4900 & 0.85 & 0.72 & 0.22 & 18.85 & 1721 & 2144 & 2204 & 2.72 \\
\hline
\end{tabular}




\section{Conclusion}

This study includes an identification of influential parameters of columns with an open and a closed cross section (RHS and U-shaped cross section) with respect to the interaction of local and global buckling. So, models of global and local stability have been created which allow an analytical solution to be used for defining the critical elastic buckling stress. The model of local buckling is created under conditions that take mutual interaction of all individual elements of the cross section into account. The model of global buckling allows a critical buckling force to be defined by means of parameters $p_{N}, \mu$ and $\psi$, taking the loss of stability and reduction of the effective zone of the cross section into account. The two dominant parameters that affect the occurrence of local and global stability of columns are related to the characteristics of the plate slenderness $t / b$ and the column slenderness $L / b$. It has been shown that the reduction in the wall thickness $t$, for example by $35 \%$ whose slenderness $t / b=0.02$, leads to a progressive decrease in the critical local buckling stress by $100 \%$. The limit slenderness of the square hollows cross section (SHS) is $t / b=0.02$, while the critical buckling load has a constant value of up to $L / b<30$. The critical buckling load decreases by reducing $t / b$. At the same time the application of the cross section with an expressed occurrence of local buckling affects the increase of the global instability of the column.

\section{Acknowledgement}

The study was financed by the Department of Traffic, Faculty of Technical Sciences in Novi Sad.

\section{REFERENCES}

[1] Degée, H.; Detzel, A.; Kuhlmann, U. Interaction of global and local buckling in welded RHS compression members. Journal of Constructional Steel Research, 64, 7(2008), pp. 755-765, https://doi.org/10.1016/j.jcsr.2008.01.032

[2] Adman, R.; Saidani. M. Elastic buckling of columns with end restraint effects, Journal of Constructional Steel Research, 87, (2013), pp.1-5, https://doi.org/10.1016/j.jcsr.2013.03.022

[3] Zhang, L.; Tong, G.S. Lateral buckling of eccentrically braced RHS columns. Thin-Walled Structures, 49 , 11(2011), pp. 1452-1459, https://doi.org/10.1016/j.tws.2011.07.006

[4] Gunalan, S.; Mahendran, M.. Improved design rules for fixed ended cold-formed steel columns subject to flexural-torsional buckling. Thin-Walled Structures, 73, (2013), pp. 1-17, https://doi.org/10.1016/j.tws.2013.06.013

[5] AS/NZ 4600: 2005. Cold formed steel structures. Australian/New Zeland Standard, 2005.

[6] AISI S 100-2007. North American specification for the design of cold-formed steel members. American Iron and Steel Institute, 2007.

[7] Schafer, B.W.; Adány, S. Buckling analysis of cold-formed steel members using CUFSM: conventional and constrained finite strip methods. Proceedings of the $18^{\text {th }}$ Int. Spec. Conf. on Cold-Formed Steel Structures, Florida, 2006, pp. 39-54

[8] Kwon, Y.B.; Kim, N.G.; Hancock, G.J. Compression tests of welded section columns undergoing buckling interaction. Journal of Constructional Steel Research, 63, 12(2007), pp. 1590-1602, https://doi.org/10.1016/j.jcsr.2007.01.011

[9] Kwon, Y.B.; Seo, E.G. Prediction of the compressive strength of welded RHS columns undergoing buckling interaction. Thin-Walled Structures, 68, (2013), pp. 141-155, https://doi.org/10.1016/j.tws.2013.03.009

[10] EN 1993-1-3, Eurocode 3 - Design of steel structures - Part 1-3: General rules - Supplementary rules for cold-formed members and sheeting, 2006.

[11] Yuan, H.X.; Wang, Y.Q.; Gardner, L.; Shi, Y.J.. Local - overall interactive buckling of welded stainless steel box section compression members. Engineering Structures, 67, (2014), pp. 62-76, https://doi.org/10.1016/j.engstruct.2014.02.012

[12] Patton, M.L.; Singh, K.D. Buckling of fixed - ended lean duplex stainless steel hollow columns of square, $\mathrm{L}, \mathrm{T}$ and + shaped sections under pure axial compression $-\mathrm{a}$ finite element study. Thin-Walled Structures, 63, (2013), pp. 106-116, https://doi.org/10.1016/j.tws.2012.09.003

[13] Bedair, O. Analytical effective width equations for limit state design of thin plates under nonhomogeneous in-plane loading. Archive of Applied Mechanics, 79, (2009) pp. 1173-1189, https://doi.org/10.1007/s00419-009-0296-z 
[14] Shanmugam, N.E.; Lakshmi, B.; Uy, B. An analytical model for thin-walled steel box columns with concrete in-fill. Engineering Structures, 24, 6(2002), pp. 825-838, https://doi.org/10.1016/S0141-0296(02)00019-6

[15] Rhodes, J. Buckling of thin plates and members - and early work on rectangular tubes. Thin-Walled Structures, 40, 2(2002), pp. 87-108, https://doi.org/10.1016/S0263-8231(01)00054-4

[16] Ellobody, E. Buckling analysis of high strength stainless steel stiffened and unstiffened slender hollow section columns. Journal of Constructional Steel Research, 63, 2(2007), pp. 145-155, https://doi.org/10.1016/j.jcsr.2006.04.007

[17] Kwon, Y.B.; Park, H.S. Compression tests of longitudinally stiffened plates undergoing distortional buckling. Journal of Constructional Steel Research, 67, 8(2011), pp. 1212-1224, https://doi.org/10.1016/j.jcsr.2011.02.015

[18] Santos, E.S.; Batista, E.M., Camotim, D.. Experimental investigation concerning lipped channel columns undergoing local-distortional-global buckling mode interaction. Thin-Walled Structures, 54, (2012), pp. 19-34, https://doi.org/10.1016/j.tws.2012.02.004

[19] Kesti, J.; Davies, J.M. Local and distortional buckling of thin-walled short columns. Thin-walled structures, 34, 2(1999), pp. 115-134, https://doi.org/10.1016/S0263-8231(99)00003-8

[20] He Z.; Zhou X.. Strength design curves and an effective width formula for cold-formed. Thin-walled structures, 79, (2014), pp. 62-70, https://doi.org/10.1016/j.tws.2014.02.004

[21] Shahabian, F.; Roberts, T.M. Buckling of slender web plates subjected to combinations of in-plane loading. Journal of Constructional Steel Research, 51, 2(1999), pp. 99-121, https://doi.org/10.1016/S0143-974X(99)00020-6

[22] Cai, J.; Long, Y.L. Local buckling of steel plates in rectangular CFT columns with binding bars. Journal of Constructional Steel Research, 65, 4(2009), pp. 965-972, https://doi.org/10.1016/j.jcsr.2008.07.025

[23] Thai, H.T.; Choi, D.H. Analytical solutions of refined plate theory for bending, buckling and vibration analyses of thick plates. Applied Mathematical Modelling, 37, 18(2013), pp. 8310-8323, https://doi.org/10.1016/j.apm.2013.03.038

[24] Djelosevic, M.; Tepic, J.; Tanackov, I.; Kostelac, M.. Mathematical identification of influential parameters on the elastic buckling of variable geometry plate. The Scientific World Journal, 2013, Article ID 268673(2013), 15 pages. https://doi.org/10.1155/2013/268673

[25] AISC Manual of Steel Construction: Load and Resistance Factor Design Specification, American Institute of Steel Construction, 3nd ed., Chicago, 2001.

[26] Djelosevic, M.; Gajic, V.; Petrovic, D.; Bizic, M. Identification of local stress parameters influencing the optimum design of box girders. Engineering Structures, 40, (2012), pp. 299-316, https://doi.org/10.1016/j.engstruct.2012.02.040

[27] Timoshenko, S.; Woinowsky-Krieger, S. Theory of Plates and Shells, 2nd ed., McGraw-Hill Book Company, New York, 1959.

[28] Timoshenko, S.P.; Gere, J.P. Theory of elastic stability, 2nd ed., McGraw-Hill Book Company, New York, 1961.

[29] Langhaar, H.L. Energy methods in applied mechanics, 2nd ed., Krieger Publishing Company, Malabar, FL: R. E., 1989.

[30] Bulson, P.S. Aluminum Federation (A.D.A), Research Report, 29, 1955.

Submitted: $\quad 29.6 .2017$

Accepted: $\quad$ 19.3.2018
Mirko Đelošević

djelosevic.m@uns.ac.rs

Ljiljana Vukajlov

vukajlov@uns.ac.rs

Goran Tepić

gtepic@uns.ac.rs

Aleksandra Milinković

a.milinkovic@uns.ac.rs

Dijana Brkljač

dijana.brkljac@uns.ac.rs

University of Novi Sad, Faculty of

Technical Sciences

Trg Dositeja Obradovića 6, Novi Sad,

Serbia 